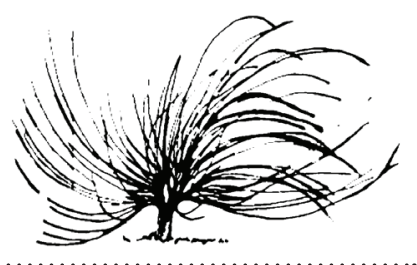

\title{
Bimodalidad, ¿necesidad u obligación para una educación a distancia democratizadora y accesible? El caso de la UNED de Costa Rica
}

\author{
Francisco Mora Vicarioli $^{1}$ \\ Universidad Estatal a Distancia de Costa Rica \\ San José, Costa Rica \\ fmora@uned.ac.cr
}

\begin{abstract}
Resumen
En este artículo se debate el uso de la bimodalidad, entendida como la posibilidad de elegir entre la educación a distancia tradicional y el uso de la virtualidad o e-learning. Esto, en momentos en que los procesos de virtualización de la Universidad Estatal a Distancia (UNED) de Costa Rica hacen que cada año se dé un uso más intensivo de este medio. Lo anterior debe ir de la mano con las posibilidades del estudiantado y las diversas facilidades que proporciona la institución, para poder brindar acceso a equipo y conectividad para la población vulnerable, así también, capacitación en el uso de las TIC que permita cerrar la brecha digital y brindar mejores oportunidades laborales. Se exponen algunos beneficios que puede brindar la bimodalidad para los procesos
\end{abstract}

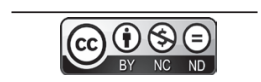

Recibido: 25 de enero de 2017-Aprobado: 3 de octubre de 2017

http://dx.doi.org/10.15359/rep.12-2.7

1 Productor académico del Programa de Aprendizaje en Línea de la Universidad Estatal a Distancia de Costa Rica. San José, Costa Rica. 
académicos, además se ofrecen algunas conclusiones y recomendaciones al respecto de la temática.

Palabras clave: educación a distancia, bimodalidad, e-learning, acceso a la educación, brecha digital, equidad.

\begin{abstract}
This article discusses the use of blended learning, understood as the possibility of choosing between traditional distance education and the use of virtuality or e-learning. This is taking place at a time when the virtualization processes of the Distance State University (UNED) of Costa Rica makes more intensive use of this resource each year. This should go hand in hand with the possibilities that students have and the various facilities provided by the institution in order to provide access to equipment and connectivity for vulnerable populations as well as training in the use of ICT to bridge the digital divide and provide better job opportunities. This article also describes some benefits that blended learning can provide for academic processes. In addition, some conclusions and recommendations are offered.
\end{abstract}

Keywords: distance education, blended learning, e -learning, access to education, digital divide, equity.

\title{
1. Introducción
}

Es deseable primero, señalar el rol de la educación a distancia y cómo esta surge con un espíritu democratizador y de acceso. De hecho, la Universidad Estatal a Distancia (UNED) tiene un modelo de acceso abierto, en cuanto a que no posee un examen de admisión en la mayoría de sus carreras y, a la vez, se tiene una gran cobertura a lo largo y ancho de nuestro país por medio de casi 40 sedes, incluso por el uso de la virtualidad tenemos la posibilidad de traspasar fronteras, por medio de la internacionalización de algunos programas.

Se debe considerar también que, la educación a distancia utiliza los medios de comunicación y en general las tecnologías de la información y comunicación (TIC) para poder cumplir su misión, hemos visto el vertiginoso avance que hemos tenido en las últimas dos décadas y 
que esto nos ha llevado al uso intensivo de la Internet, los contenidos digitales y las diversas plataformas de aprendizaje en línea, con lo cual nace la educación mediada por la virtualidad, más conocida en el ámbito internacional como e-learning, como una expresión "moderna" o actualizada de la educación a distancia, recordemos que esta última hace uso de las tecnologías conforme estas van surgiendo.

La UNED de Costa Rica cumple en el 2017, 40 años como institución pionera en el país y en la región en cuanto a la modalidad a distancia. La evolución de las tecnologías de la información y comunicación ha impulsado grandes cambios que han supuesto el uso del e-learning, si bien la UNED es una institución a distancia y no virtual, las estadísticas en el uso de las plataformas de aprendizaje en línea dejan entrever que la tendencia es hacia una virtualización de las diferentes carreras y programas. En el 2016 se tuvo la apertura de 1877 entornos virtuales de cursos y asignaturas, así como 85590 usuarios (PAL, 2016).

Tal como lo aporta Vargas (2014), refiriéndose al papel de la educación superior y en particular la estatal:

La universidad pública tiene un doble rol, pues además de la formación académica que debe ofrecer para responder a las transformaciones sociales, tiene un compromiso con las poblaciones más necesitadas, ya que la inversión estatal que la hace posible, debe impactar a estas poblaciones; ello suscita una relación polémica en cuanto al acceso, pues este también está condicionado por la calidad académica del estudiantado; calidad que también es determinada en gran medida por la calidad de la educación preuniversitaria a la que se pudo tener acceso; situación ante la cual la condición socioeconómica es un determinante importante en el tipo de institución educativa a la que se accede (p. 15).

La UNED, como institución de educación superior estatal, tiene el reto de brindar acceso y calidad académica, pero sin dejar de lado las características de la población que debe atender, pues nace con una misión y visión particulares, que a continuación se enuncian: 


\begin{tabular}{|c|c|}
\hline Misión & Visión \\
\hline $\begin{array}{l}\text { La Universidad Estatal a Distancia } \\
\text { (UNED) es una institución de carác- } \\
\text { ter público que goza de autonomía. Su } \\
\text { misión es ofrecer educación superior } \\
\text { a todos los sectores de la población, } \\
\text { especialmente a aquellos que, por ra- } \\
\text { zones económicas, sociales, geográfi- } \\
\text { cas, culturales, etarias, de discapaci- } \\
\text { dad o de género, requieren oportuni- } \\
\text { dades para una inserción real y equita- } \\
\text { tiva en la sociedad (UNED, s.f.). }\end{array}$ & $\begin{array}{l}\text { La UNED será líder en los procesos } \\
\text { de enseñanza y aprendizaje a distan- } \\
\text { cia que emplean de manera apropia- } \\
\text { da y mediados pedagógicamente, } \\
\text { tecnologías y otros medios de comu- } \\
\text { nicación social (UNED, s.f.). }\end{array}$ \\
\hline
\end{tabular}

Es por lo anterior que la UNED tiene el reto por un uso responsable de las TIC que garanticen el cumplimiento de los fines de su creación.

\section{Objeto de estudio}

El objetivo de este artículo es: analizar el rol de la bimodalidad en la educación a distancia. Para cumplir este propósito, a continuación, se desarrollan de manera crítica ciertas temáticas relativas a este.

\section{Metodología}

Como parte de la metodología de este artículo, se procedió a realizar una precisión del tema de la bimodalidad, por medio de una búsqueda bibliográfica, mediante el uso de fuentes bibliográficas actualizadas sobre la temática, tanto en idioma español como en inglés. Por otra parte, se utilizó un diseño cualitativo, para contar con criterio de fuentes de primera mano, por parte de académicos de la UNED que aplican la bimodalidad dentro de los cursos y las asignaturas a cargo, así como otros expertos con experiencia en la gestión curricular y aprendizaje en línea. Se aplicó una entrevista conformada por preguntas abiertas sobre la temática, la información generada sirvió de sustento para la discusión teórica y la validez por medio de una triangulación del análisis realizado, esto basado en las recomendaciones de Nathan (2011), en un estudio sobre el e-learning. Según Hernández, Fernández y Baptista (2010), en relación con la técnica de la entrevista, señalan 
que: "el investigador cualitativo utiliza técnicas para recolectar datos, como la observación no estructurada, entrevistas abiertas, revisión de documentos (...)" (p. 9).

\section{Educación a distancia democratizadora}

Cabero (2016), refiriéndose a la evolución de la educación a distancia, señala que:

Ha dado un salto cualitativo significativo en las últimas décadas, cuando ha pasado de ser considerada una educación de segundo orden y con una imagen social poco prestigiosa a ser contemplada como una educación con altas potencialidades, además, bastante deseada; ello independientemente del país al que nos refiramos o el nivel educativo en que se concrete. Creo que no me equivoco al afirmar que en estos momentos nos hallamos con una paradoja y es que los mismos detractores, personas e instituciones que ponían en duda su validez, invierten importantes recursos y esfuerzos en su desarrollo y la comienzan a defender con bastante avidez (p. 1).

Para Ramírez y Rama (2014), la educación a distancia "surge como un modelo de educación inclusiva, que da oportunidades a personas que por su condición social, laboral o familiar no tienen las mismas oportunidades para estudiar en modelo de educación presencial" ( $p$. 175). Es así como, a través de la implementación de diversos medios y recursos educativos es posible llegar a quienes buscan superarse a pesar de sus condiciones socioeconómicas o de acceso geográfico, pero que de otra manera no sería posible.

Hoy, con el uso de las TIC, el modelo a distancia ha sufrido transformaciones para buscar integrar herramientas y materiales educativos digitales que dinamicen los procesos de aprendizaje, sin dejar de lado el propósito para el cual está al servicio de la sociedad. Al respecto, Domínguez y Rodríguez (2013) destacan que:

Las tendencias en la educación se orientan hacia la integración de las modalidades de estudio que faciliten el diseño de las asignaturas con estrategias de modalidades presencial, distancia y 
virtual, a fin de que el estudiante sea protagonista del proceso enseñanza-aprendizaje de calidad con la diversidad de medios. Esta integración de las modalidades ha de ser expresión no solo del uso intensivo de la TIC, sino del cambio de paradigma, de una cultura y de formas de gestión (p. 50).

Es así como, la educación a distancia ha evolucionado de manera significativa desde su origen al brindar mayores posibilidades de interacción que le permiten al estudiante involucrarse más ampliamente en su proceso de aprendizaje; gracias al uso de los recursos tecnológicos es posible ampliar el acceso y los medios de comunicación, lo cual repercute en el acompañamiento que se le pueda brindar al estudiantado y la interacción que este último puede tener con otros pares.

\section{Educación a distancia mediada por la virtualidad}

En el modelo de educación a distancia, los sistemas de gestión de aprendizaje (o LMS por sus siglas en inglés, Learning Managment System) o plataformas de aprendizaje en línea son el soporte tecnológico que permite la interacción entre docentes y estudiantes, así como la incorporación de contenidos y actividades didácticas en los cursos.

Es a través del uso de dichos sistemas que la educación apoyada por la virtualidad se está implementando no solo en las instituciones educativas con modalidad a distancia, sino también en aquellas con modalidad presencial para así facilitar el acceso a todo tipo de poblaciones estudiantiles. Al respecto, Arboleda y Rama (2013) comentan que:

La educación virtual adquiere cada día mayor valor pedagógico para potenciar un aprendizaje de calidad y pertinencia, con equidad e inclusión social al que puedan acceder no solo los estudiantes regulares, los de las regiones apartadas, con limitaciones tecnológicas y culturales, sino también quienes están en situación de discapacidad (p. 59).

A partir de ello, surgen también nuevas opciones y posibilidades para el desarrollo de materiales educativos que apoyen el proceso educativo a través de los LMS. Sobre esto Rama (2012) orienta que: 
Las plataformas digitales impactaron en los recursos de aprendizaje gracias a la convergencia tecnológica y facilitaron el pasaje desde materiales instruccionales planos hacia recursos digitales en red, tales como el hipertexto y la confluencia de audio, texto, video y programación para guiar y apoyar los procesos de aprendizaje. Los modelos de simulación o los programas de carácter lúdicos orientados al aprendizaje, se articulan, además, a concepciones más modernas sobre el papel de la experimentación como mecanismo de aprendizaje más eficiente (p. 132).

Al considerar lo anterior, no es de extrañar que dicha modalidad de estudio sea ampliamente considerada como una de las mejores opciones para optar por un título de educación superior. Y es que la educación a distancia se va perfilando como un nuevo escenario a contemplar por la aceptación en crecimiento que los estudiantes han encontrado en dicha modalidad de estudio, al ofrecerles una oportunidad de profesionalizarse manteniendo los estándares de calidad esperados (Domínguez y Rodríguez, 2013).

Por lo tanto, es comprensible que cada año la demanda aumente y más universidades muestren interés en desarrollar e implementar sistemas $\mathrm{y}$ herramientas que les permita gestionar el aprendizaje de manera virtual.

Para Ileana Salas Campos (2016), el e-learning se puede definir en forma básica como: "aprendizaje electrónico, aquel que es producto de un diseño didáctico soportado en el uso de tecnologías digitales" (comunicado personal, 11 de noviembre del 2016). De manera complementaria, Pérez, López, Romero y Urquía (2016), proponen el e-learning de la siguiente forma: "un proceso de enseñanza aprendizaje apoyado en la red, caracterizado por la separación física entre profesorado y alumnado, facilitando la comunicación síncrona y asíncrona, llevándose a cabo una interacción didáctica continuada" (p. 12).

El término e-learning es cada vez más conocido en el ámbito educativo, pues se considera una opción flexible y accesible a la población, por cuanto rompe con las barreras de tiempo y espacio, a la vez que los dispositivos inteligentes colaboran con la conectividad necesaria para que se lleve a cabo. Tal como lo aporta García (2016), concibe el e-learning delimitándolo de la siguiente manera: 
Su principal característica es que el facilitador y el estudiante no coinciden en un mismo espacio físico ni en el mismo horario, es decir, todo el proceso de enseñanza-aprendizaje se realiza a través de una computadora con conexión a Internet (asincrónico) (p. 14).

En la región existe un importante desarrollo en el campo del e-learning y la educación que utiliza de manera parcial la virtualidad, tal como lo aporta Rama (2013):

En América Latina se está avanzando diferenciadamente en los procesos de digitalización y virtualización de la educación superior. En ella se constata globalmente la convergencia tecnológica y la lenta desaparición de la dualidad de modalidades, como resultado de los diversos caminos de la virtualización y digitalización. Se verifica un mayor avance a modelos de "blended learning" o educación semivirtual, que se gesta desde el paradigma a distancia o del paradigma presencial. Ambos sin duda tienen sus propias eficacias y limitaciones en términos educativos para contribuir a adquirir competencias (p. 23).

Cabe destacar que las instituciones de educación presencial ya utilizan el e-learning como recurso de apoyo, dados los beneficios; paulatinamente existe mayor reconocimiento de la validez y pertinencia del e-learning y, por consiguiente, de la educación a distancia.

Según el Ministerio de Educación Nacional de Colombia:

La educación virtual, también llamada "educación en línea", hace referencia al desarrollo de programas de formación que tienen como escenario de enseñanza y aprendizaje el ciberespacio, en el cual no es necesario que se dé un encuentro cara a cara entre el profesor y estudiante, sin embargo, es posible establecer una relación interpersonal de carácter educativo (Pubiales y de Fonseca, 2014, p. 4).

Para que pueda ocurrir el e-learning se requiere de una plataforma tecnológica, este tipo de programas tienen funcionalidades comunes para la gestión del aprendizaje, existen algunas de código abierto o de 
software libre, así como privativas o que implican el pago de un servicio y licenciamiento, esto es aclarado por Onrubia (2016):

Un entorno virtual de enseñanza y aprendizaje puede disponer de herramientas de trabajo en equipo para el aprendizaje y la construcción de conocimiento y, en cambio, estas herramientas al no ser utilizadas efectivamente por los participantes, o emplearse estrictamente como herramientas de comunicación, pero no como herramientas de colaboración. O inversamente, un determinado tipo de actividades puede no estar previsto en el diseño pedagógico, pero acabar desplegándose espontáneamente en el desarrollo del proceso de enseñanza y aprendizaje (p. 6).

Según Illera (2013): “en estos últimos diez años, la tecnología y su impacto social han ido muy por delante de los modelos pedagógicos imperantes, que se han considerado poco adecuados para una realidad tan cambiante" (p. 4). Como vemos, el avance tecnológico viene a impactar en los procesos educativos y la forma en que se llevan a cabo.

Finalmente, para Fermoso y Pedrero (2009), citados por Pubiales y de Fonseca (2014):

Las nuevas tecnologías y los medios digitales plantean nuevas posibilidades educativas, dando paso a lo que se conoce como e-learning. Sin embargo, contar con estos medios no garantiza sin más el conseguir los mejores resultados de aprendizaje, pues también se requiere la implantación paralela de nuevos modelos pedagógicos. Estos modelos afectarán tanto al diseño de los materiales educativos en formato digital, como al modo de utilización de los mismos en el aula (p. 3).

La visión de los autores anteriores es clara en que las tecnologías, y en particular el uso del e-learning, no garantiza el éxito, pues el modelo pedagógico debe actualizarse en función de este y el tipo de recursos que se utilizarán.

Cabe señalar que existen múltiples referentes sobre estudios recientes en cuanto al aporte del e-learning y sus beneficios, así también en lo relativo al rendimiento académico, realizados por: Tilve, Núñez y Fernández (2013), Rodríguez, Gómez y Ariza (2014), Gutiérrez y 
García (2016), Genc, Kulusakli y Aydin (2016) y el de Andresen (2016), si bien los estudios se enfocan en diferentes aristas, el común denominador son los aportes del e-learning en cuanto a la mejora del acceso, comunicación e interacción, así también algunas variantes o expresiones más recientes del uso de la virtualidad.

\section{El tutor facilitador de cursos en línea}

La función tutorial en los cursos en línea la debe asumir un profesional experto en el contenido, con ciertas competencias tecnológicas que le permitan el uso del recurso virtual de manera apropiada, así como pedagógicas, para una adecuada mediación del proceso de enseñanza. Otros requisitos deseables serían la experiencia en educación a distancia mediada por la virtualidad y la capacitación en tutoría virtual, sin embargo, dichos aspectos no necesariamente los puede cumplir la totalidad del profesorado o por lo menos en la UNED de Costa Rica aún no ocurre, pero la institución educativa debe proveer los espacios de formación y asesoramiento flexibles que le permitan al tutor desarrollar las competencias necesarias para su óptimo cumplimiento, así también mecanismos de seguimiento a su desempeño en dicho escenario.

De acuerdo con Gamboa (2013), algunas funciones del tutor virtual son:

- Diseñar contenidos y actividades que fomenten el desarrollo de competencias, el pensamiento crítico, el trabajo en equipo, la autorregulación y la autonomía de sus estudiantes.

- Utilizar variedad de técnicas y estrategias innovadoras que garanticen el desarrollo de materiales que puedan adaptarse, según la tecnología utilizada, a los diversos estilos de aprendizaje.

- Evaluar el aprendizaje en forma integral, pertinente y coherente con los objetivos de aprendizaje propuestos.

- Utilizar la tecnología como un medio que promueva la comunicación y la interacción dentro de los procesos de enseñanza y aprendizaje.

- Buscar espacios de capacitación que le permitan mantenerse informado y actualizado en su disciplina (p. 10). 
De las funciones anteriores se puede evidenciar la complejidad del quehacer del tutor, pues en la modalidad a distancia donde media la tecnología se incrementa la labor comunicativa, a la vez que la dimensión evaluativa se puede llevar a cabo de manera más intensa, particularmente en cuanto al diagnóstico y lo formativo, por los espacios de realimentación de que disponen las plataformas de aprendizaje en línea.

Con respecto a la atención de cursos en línea, la responsabilidad de un tutor o facilitador es grande en cuanto a las funciones que debe asumir, y el hecho de comprender adecuadamente los roles permite la correcta ejecución, mediación y facilitación de un curso en línea.

\section{TIC y derecho a la educación}

Las TIC al ser un instrumento que puede mejorar el acceso a la educación y apoyarla, van de la mano con el artículo 26 de la Declaración Universal de los Derechos Humanos (1948), donde tenemos la visión de la educación como nuestro derecho, pero no es solo para que las personas tengan un determinado nivel de instrucción, sino que reciban una educación específica para que se logre alcanzar el pleno desarrollo de la personalidad, el respeto por los derechos humanos y las libertades fundamentales. Debemos tener en cuenta que, como parte del perfil de salida de la mayoría de profesionales, las competencias en el uso de las TIC son necesarias, de lo contrario estarán en desventaja laboral. Por ello, estos son ejes transversales, muchas veces no declarados, pero que exigen al estudiante aprender a desempeñarse en el uso de paquetes ofimáticos, programas y recursos en Internet, comunicación digital, entre otros.

Por medio de las tecnologías digitales y su uso en la educación a distancia, logramos propiciar la adaptabilidad: "la educación ha de tener la flexibilidad necesaria para adaptarse a las necesidades de sociedades y comunidades en transformación y responder a las necesidades de los alumnos en contextos culturales y sociales variados" (ONU, 1999, p. 3). Si negamos el uso de las TIC estamos limitando a la población que tiene acceso, evitamos también, como se mencionó, que se desarrollen competencias valiosas en cuanto al uso de estas, que servirán a los futuros profesionales. Acá puede apoyar también la visión de Delors (1999): “(...) debe imponerse el concepto de educación durante toda la vida con sus ventajas de flexibilidad, diversidad 
y accesibilidad en el tiempo y el espacio" (p. 14). En este sentido, si existe un modelo educativo que facilita la autonomía y autorregulación, es la educación a distancia, esto favorece que el individuo desarrolle competencias muy valiosas, sin dejar de lado al e-learning como expresión de la educación a distancia.

\section{Educación a distancia e inclusiva y accesible}

La educación a distancia por su naturaleza propicia la inclusión, para Cabero (2016):

Hablar de inclusión educativa, es no caer en el error de pensar que sus destinatarios son los sujetos con algún tipo de discapacidad. Hablar de inclusión educativa es referirnos al derecho que tienen todas las personas a la educación, y que son excluidas del sistema educativo por aspectos como el género, la cultura, la raza o sus características personales (p. 2).

Cabe señalar que la UNED de Costa Rica, tiene instancias de apoyo para que esta inclusividad se lleve a cabo, tanto para poblaciones con escasos recursos, población privada de libertad, así como para atender las discapacidades.

Tal como lo señala Sánchez (2013), la inclusividad en el e-learning se puede propiciar cuando se tiene accesibilidad, que se define como:

La accesibilidad ha de ser entendida en toda su amplitud, incidiendo en la creación de espacios inclusivos donde todas las personas puedan relacionarse de forma sencilla, sabiendo que las medidas a diseñar y desarrollar han de ser evaluadas desde la comprensión de la discapacidad como punto de encuentro entre el entorno, que desempeña un papel fundamental, y las características personales del individuo (p. 6).

Según la perspectiva anterior, la UNED se orienta hacia un diseño inclusivo, por medio del diseño universal para el aprendizaje (DUA), el cual se concibe como: "herramienta metodológica orientada a flexibilizar el currículo considerando la diversidad de necesidades de aprendizaje del estudiantado y sus habilidades sensoriales, 
motrices, cognitivas, afectivas y lingüísticas" (UNED, 2017, p. 116). Este principio está permeando la elaboración del material didáctico, lo cual beneficia en el uso de la virtualidad, pues esta última es un espacio de convergencia para los contenidos, en los diferentes formatos, texto, audio, video, multimedia, entre otros. Cabe señalar también que existen en la UNED grupos colaborando con el asesoramiento de las prácticas del diseño universal, para permear las prácticas en producción de materiales didácticos que favorezcan la adaptabilidad a las diversas poblaciones y estilos de aprendizaje.

\section{Brecha digital y acceso a las TIC}

El tema de la brecha digital y acceso tiene complejidad en cuanto a su abordaje, pues el hecho de contar con equipo no garantiza que exista condición para el uso de las TIC, ambos conceptos están ligados. Para Gros y Contreras (2006), citados por Muñoz y Nicaragua (2014):

La alfabetización digital es mucho más compleja y está compuesta, no solamente por un conjunto de habilidades sociocognitivas necesarias para utilizar adecuadamente las tecnologías, sino también por otras como: tener un pensamiento crítico y realizar juicios de valor informados acerca de la información digital; utilizar destrezas de lectores y de comprensión de hipertextos dinámicos y no secuenciales, así como destrezas de construcción del conocimiento a partir de información confiable, sin sesgos y de diversas fuentes, entre otros (p. 11).

Bajo la visión amplia, en que lo digital no es solo un medio, sino un espacio de interacción, abordado de manera crítica es que la alfabetización digital cobra mayor sentido. Complementariamente a lo anterior, desde la óptica de Cruz (2014):

La desigualdad tecnológica no solo tiene que ver con las posibilidades disímiles de acceder a la conexión sino con su efecto sobre la vida social y económica de las personas. Si una persona sabe utilizar esa conexión a Internet para hacer comercio electrónico en su empresa y otra solo la utiliza para revisar el correo o entrar a Facebook, esa diferencia incide en el tamaño de la brecha (párr. 2-3). 
La consideración que nos brinda el autor es clave para identificar la brecha digital que existe en la población estudiantil, este puede ser un filtro que oriente a la aplicación y pertinencia de la bimodalidad, como oferta en que el estudiante elige la modalidad en que llevará a cabo su proceso de formación.

Además de lo anterior, Delors (1999) orienta que:

Mientras la sociedad de la información se desarrolla y multiplica las posibilidades de acceso a los datos y a los hechos, la educación debe permitir que todos puedan aprovechar esta información, recabarla, seleccionarla, ordenarla, manejarla y utilizarla. Por consiguiente, la educación tiene que adaptarse en todo momento a los cambios de la sociedad, sin dejar de transmitir por ello el saber adquirido, los principios y los frutos de la experiencia (p. 18).

Ciertamente, la educación es la llave por la cual podemos potenciar el uso de las TIC en favor de los procesos académicos, como un medio no como un fin.

En cuanto a los datos nacionales, Muñoz y Nicaragua (2014) aportan que:

La Encuesta Nacional de Hogares 2011 realizada por el Instituto Nacional de Estadística y Censos (INEC) a 1.297.522 viviendas, destaca algunos datos relativos a la conectividad en nuestro país. En cuanto a la tenencia de computadoras, se puede observar que el 45,3\% de los hogares de la muestra cuenta con este dispositivo y que un 33,6\% tiene conexión a Internet en casa. Eso significa que, de las personas que tienen las plataformas para acceso a Internet, un $11,7 \%$ no tiene esta conectividad (p. 9).

Las cifras anteriores resultan alentadoras, incluso para la época en que se realizó el censo, el acceso resulta positivo y más aún sorprende el crecimiento vertiginoso. 


\section{Apoyos para el acceso y cierre de la brecha digital}

La UNED ha tratado el tema del apoyo en cuanto al acceso, de hecho, posee el Plan de Desarrollo Académico (2012-2017) donde brinda algunos lineamientos, estos van de la mano con las Políticas Institucionales de Desarrollo 2012-2017, que enuncian lo siguiente:

Las TIC ocupan un papel importante para potenciar los procesos de regionalización e internacionalización, la garantía de la cobertura, la equidad, la pertinencia y la calidad académica. Para ello se propone la inversión estratégica, en infraestructura física y tecnológica, que potencie el modelo de educación (UNED, 2012, p. 31).

Como se puede notar de lo anterior, existe un respaldo en cuanto a la inversión que se debe hacer para continuar con un modelo apoyado por las TIC. Luego de un análisis del Plan de Desarrollo Académico (2012-2017), y en particular en el eje de las TIC que contiene, se debió brindar mayor importancia para tratar aspectos de acceso y brecha digital, pues no trascienden de su mención.

Entre las conclusiones del Plan de Desarrollo Académico (20122017) y contextualizados el eje de las TIC, se destaca que:

Para los grupos menos favorecidos: “(...) la universidad tiene la responsabilidad de dar el acceso y la capacitación debida a estos grupos" (...). Por otra parte, “(...) atención a la diversidad. Garantizar acceso y cobertura a todos los estudiantes (...)" (UNED, 2012, p. 53).

Una aclaración importante que se hace corresponde a: “(...) las TIC no son solo cursos en línea y deben plantearse en función de todo lo que engloban. El Plan de Desarrollo Académico tiene que contemplar el diseño de todos los medios (no solo plataformas)" (UNED, 2012, p. 53).

Un punto importante que reconoce expresamente el Plan es: "Falta de investigaciones en el área de TIC y discapacidad enfocada a estudiantes universitarios" (UNED, 2012, p. 53). Esta conclusión permite tener en cuenta el trabajo en el campo de las personas con discapacidad y sobre todo brindar materiales y plataformas con un diseño accesible y universal. 
Otra medida importante es que desde el 2012 se trabaja fuertemente para contar con una red inalámbrica o Wifi tanto en la sede central como en las casi 40 sedes de la UNED, que permita a los estudiantes contar con este importante recurso, además de laboratorios de cómputo con posibilidad de acceso en algunos de estos centros por medio de franjas horarias. También, en el Programa de Aprendizaje en Línea, desde su creación, hace más de 10 años, en cada inicio de periodo académico, se brindan insumos operativos o técnicos para los estudiantes que tendrán cursos en línea o con apoyo virtual, asimismo se brinda soporte técnico por diferentes vías: presencial, telefónica, correo electrónico y redes sociales. Lo anterior es un apoyo que se le brinda a la población que puede experimentar dificultades técnicas, en particular para estudiantes de primer ingreso, que se enfrentan a la modalidad a distancia y virtual.

\section{La bimodalidad}

La bimodalidad, tal como la define e implementa la UNED de Costa Rica, consiste, al menos, en los programas que aún la mantienen, en la posibilidad de que el estudiantado seleccione la modalidad en que desea llevar su proceso educativo: si a distancia tradicional, que aún se concibe como aquella en la que no se utiliza la virtualidad, donde mayoritariamente se utilizan más que todo recursos impresos, así como recursos digitales soportados de manera física como discos compactos; en cuanto a las actividades, se mantiene la aplicación de exámenes de manera presencial, así como la entrega física de tareas. También, tenemos la opción que sea por medio de la virtualidad o el uso de las plataformas de aprendizaje en línea.

Tal como lo aporta la Vicerrectoría Académica de la UNED (2011), refiriéndose a la bimodalidad, que es donde:

El estudiante tiene la posibilidad de matricular una asignatura o curso que se oferta en dos modalidades: la tradicional a distancia o con apoyo de la virtualidad, por ello, la forma en que se ofertará debe ser de conocimiento de los estudiantes desde el momento de la matrícula. Es importante indicar que en una asignatura o curso que cumpla esta característica no variarán los objetivos y los contenidos, pero sí se requiere hacer una diferencia en la propuesta metodológica; por tanto, es necesario contar con un 
diseño curricular para cada opción, así como con su respectiva orientación académica (citado por la UNED, 2017, p. 76).

Lo anterior deriva en las siguientes preguntas de interés para este artículo y cuyas respuesta o acercamiento a estas, podrían justificar la pertinencia de la bimodalidad:

1. ¿La bimodalidad apoya la misión y visión de la UNED?

2. ¿Qué retos presenta la bimodalidad para las cátedras y programas de la UNED?

3. ¿Cuáles son los beneficios de la bimodalidad?

Las respuestas a las preguntas anteriores no son sencillas, de hecho, no han sido abordadas por la UNED de manera formal por medio de procesos investigativos, sino que se ha seguido una tendencia de virtualización y eliminación de la modalidad a distancia tradicional, pues la primera tiene una serie de ventajas de comunicación, interacción, convergencia de contenidos, entre otras. En tanto la educación a distancia tradicional se perfila como una modalidad donde el estudiante aprende los contenidos y realiza las actividades de manera independiente.

Para la doctora Ileana Salas Campos, especialista en tecnología educativa: la bimodalidad no favorece la misión y visión de la UNED,

Sino en la flexibilidad que podamos brindar en la oferta académica, se debe tener en cuenta que la bimodalidad demanda una doble planificación de la asignatura, y lógicamente, un doble modelo evaluativo, lo cual podría representar una carga de trabajo extenuante para muchas cátedras y programas (comunicado personal, 16 de marzo de 2017).

La flexibilidad y adaptabilidad de la oferta son las que pueden aportar que se cumpla con la misión democratizadora de la educación superior. El hecho de contar con bimodalidad en los cursos de las carreras supone contar con dos ofertas distintas respecto a diseño, planeación y atención, pues las modalidades a distancia tradicional y virtual, son muy diferentes en cuanto a posibilidades.

Desde la perspectiva de la máster Cinthya Valerio Álvarez, productora académica y asesora de asignaturas en línea de la UNED, 
tenemos beneficios de la bimodalidad como: "posibilidad de elección por parte del estudiantado, acorde con las posibilidades laborales y de acceso" (comunicado personal, 20 de marzo del 2017). En cuanto a los retos que representa esta modalidad, según Valerio se señalan: "desde la dimensión administrativa implica una doble planificación de las asignaturas y asignación de actividades diferentes de la mano con modelos de evaluación distintos" (comunicado personal, 20 de marzo del 2017).

Por otra parte, el máster Christian Ocampo Hernández, encargado de la Cátedra de Métodos de Estudio a Distancia e Investigación, señala que la bimodalidad: "debe ser el camino a seguir, ya que brinda dos posibilidades a los estudiantes: la de cursar la asignatura en modalidad regular o bien, en modalidad virtual, por lo que brinda cierta flexibilidad" (comunicado personal, 13 de marzo del 2017).

Se debe considerar también que, el hecho de contar con docentes capacitados para trabajar en los entornos virtuales de aprendizaje no es sencillo, pues la mayoría de profesionales son formados en la presencialidad, lo cual implica que generarán sus primeras experiencias en la educación a distancia y virtual, cuando inicien sus labores en la UNED.

Sobre la capacitación, cabe destacar que la UNED cuenta con un Centro de Capacitación en Educación a Distancia (CECED), el cual tiene una oferta base de capacitación para el profesorado que utilizará los entornos virtuales de aprendizaje. Algunas ofertas se desarrollan completamente en línea, otras de manera híbrida, que se refieren a que disponen de sesiones de asistencia presencial combinadas con el uso de la virtualidad. Se generan ofertas normalmente cuatrimestrales. Es por lo anterior, que se cuenta con opciones de capacitación para el cuerpo docente, pero de acuerdo con la perspectiva del análisis de este ensayo, estas deben ampliarse en cuanto a cobertura y flexibilidad en la capacitación, diferentes horarios y modalidades de talleres cortos, que respondan a la población de tutoras que es una característica de la UNED.

En cuanto a la mediación de los entornos y en general del uso de la virtualidad, cabe señalar que la institución ha sumado esfuerzos en relación con la creación de un instrumento de criterios de calidad, por medio de una comisión. Durante el 2017 se hará el primer pilotaje al respecto, con el fin de contar con mecanismos institucionales formales de la verificación de la calidad de los cursos en línea. Lo anterior va de la mano con los procesos de autoevaluación y acreditación en que ya están inmersos muchos programas de estudio. 
La institución cuenta con el Programa de Aprendizaje en Línea (PAL), instancia de soporte y asesoramiento para el uso de los entornos virtuales de aprendizaje, que colabora con la inducción y apoyo a estudiantes, por diferentes vías, telefónica y electrónica, así también se hace la atención del profesorado en cuanto al uso técnico y pedagógico de las plataformas, tanto de manera presencial como a distancia. Dadas las necesidades del estudiantado, se deberían ampliar los horarios y los días de atención con respecto al soporte técnico dirigido a este.

\section{Conclusiones y recomendaciones}

Desde la perspectiva de que la UNED es una institución a distancia y no virtual, resulta complejo virtualizar los diferentes programas y carreras, sin contar con estudios sobre el acceso del estudiantado tanto de equipo como de conectividad.

La bimodalidad, es decir, el hecho de elegir la modalidad puede apoyar el espíritu democratizador de la educación con que surge la educación a distancia. Para tal efecto, es deseable contar con investigaciones que señalen el perfil del estudiante y sus condiciones de acceso a la tecnología, así como las destrezas al respecto.

Según los consultados es la flexibilidad que brinda la bimodalidad lo que da el mayor aporte al estudiantado al estar más acorde con sus necesidades. Sin embargo, esta modalidad implica un reto para la institución, pues deberá contar con una doble planificación de las asignaturas.

Cabe señalar que la bimodalidad no es actualmente considerada como obligatoria, sino que algunas cátedras aún la mantienen por convencimiento de que es una forma de brindar mejores opciones para el estudiantado. Algunas cátedras aplican esta modalidad a manera de plan piloto para valorar la pertinencia y resultados de esta.

Si la UNED apuesta por la virtualidad, debe contar con el apoyo suficiente para las poblaciones más vulnerables, de lo contrario no se cumpliría con su misión y visión. A propósito de esto, existen estrategias que desarrolla la institución para poder brindar apoyo a estas poblaciones, ya que también se debe limitar la brecha en el uso de las tecnologías.

La UNED debe divulgar a su población, en particular para los que la consideran como una opción de educación superior, el uso de la virtualidad que tendrán en los diferentes programas de pregrado, grado y posgrado, así también los requerimientos técnicos y de acceso que se tendrán. 
Los datos de acceso a Internet en la población, según las últimas investigaciones nacionales pueden orientar sobre la pertinencia del uso de la modalidad virtual, aunque como no se tiene un acceso del 100\% de la población, no existe garantía que las poblaciones más vulnerables tengan la facilidad para acceder a la educación a distancia, por ello la bimodalidad podría permitir mayor equidad.

La UNED cuenta con mecanismos de capacitación docente y soporte técnico que favorecen la bimodalidad.

Para futuras investigaciones, sería adecuado incluir en los procesos de investigación, la atención al tema de la bimodalidad y el impacto en programas que así lo implementan, asimismo revisar si existe impacto en la mejora del rendimiento académico de los estudiantes, comparando los rendimientos para ambas modalidades. Algunas preguntas que se mantienen abiertas en torno a este debate son:

1. ¿Todos los estudiantes de la UNED tienen las posibilidades de acceso a equipo de cómputo e Internet?

2. ¿Existen preferencias marcadas entre la modalidad tradicional a distancia y la virtual?

3. ¿Cuenta la UNED con estrategias para apoyar el acceso y cierre de brecha digital para poblaciones vulnerables?

\section{Referencias}

Andresen, B. B. (2016). Enhanced self-efficacy and blended e-learning -- mixed innovations. Annual International Conference On Education \& E-Learning, 28-33. doi:10.5176/2251-1814_EeL16.10. Recuperado de http://search.ebscohost.com/login.aspx?direct $=$ true \& $\mathrm{db}=$ eue \&AN=118465632\&lang=es\&site=ehost-live

Arboleda, N. y Rama, C. (2013). La educación superior a distancia y virtual en Colombia: nuevas realidades. Recuperado de http:// virtualeduca.org/documentos/observatorio/la_educacion_superior_a_distancia_y_virtual_en_colombia_nuevas_realidades.pdf

Cabero, J. (2016). La educación a distancia como estrategia de inclusión social y educativa. Recuperado de https://idus.us.es/xmlui/ handle/11441/34247

Cruz, M. (16 de noviembre de 2014). Costa Rica mide brecha digital con métodos insuficientes. Recuperado de http://www.elfinancierocr. 
com/tecnologia/brecha_digital-telefonia_movil-medicion_de_ brecha-datos-indicadores_0_628737181.html

Delors, J. et al. (1999). La educación encierra un tesoro. Madrid: UNESCO-Santillana, Paris. Recuperado de http://www.unesco. org/education/pdf/DELORS_S.PDF

Domínguez, J. y Rodríguez, J. (Eds.) (2013). La educación a distancia en el Perú. Recuperado de http://virtualeduca.org/documentos/ observatorio/la_educacion_a_distancia_en_peru.pdf

Gamboa, Y. (2013). La tutoría virtual, quehaceres para el buen desempeño. Congreso Edutec-UNED. San José, Costa Rica.

García, F. (2016). Diseño de un modelo curricular E-learning, utilizando una metodología activa participativa. RIDE Revista Iberoamericana para la Investigación y el Desarrollo Educativo, 7(13), 147182. Recuperado de http://www.ride.org.mx/index.php/RIDE/ article/view/240

Genc, G., Kulusakli, E. y Aydin, S. (2016). A Comparative Study on the Motivation and Attitudes of Language Learners of Online Distance and Traditional In-Classroom Education. Turkish Online Journal Of Distance Education, 17(4), 63-75. Recuperado de http://search.ebscohost.com/login.aspx?direct=true\&d$\mathrm{b}=$ eric\&AN=EJ1116776\&lang $=$ es\&site $=$ ehost-live

Gutiérrez, R. y García, A. (2016). ¿Cómo mejorar la calidad, la motivación y el compromiso estudiantil en la educación virtual? (Spanish). Campus Virtuales, 5(1), 74-82. Recuperado de http://search.ebscohost.com/login.aspx?direct=true $\& \mathrm{db}=$ eue\&AN=114762071\&lang=es\&site=ehost-live

Hernández, R., Fernández, C. y Baptista, P. (2010). Metodología de la investigación. Quinta Edición. México: MacGraw Hill.

Illera, J. (2013). Enseñar y aprender en entornos digitales. Investigación y ciencia, 444, 47-51. Recuperado de http://portafoli.ub.edu/portfolios/jlrodriguez/4571/last/media/09-13-rdezillera.pdf

Muñoz, M. y Nicaragua, R. (2014). Un acercamiento a la brecha digital en Costa Rica desde el punto de vista del acceso, la conectividad y la alfabetización digital. Recuperado de http://revistas.ucr.ac. cr/index.php/eciencias/article/view/12866/12328

Nathan, E. (2011). Critical Success Factors: How One Multinational Company Develops Global E-Learning. Performance Improvement Quarterly, 24(1), 7-30. Recuperado de http://search. 
ebscohost.com/login.asp $\mathrm{x}$ ?direct $=$ true $\& \mathrm{db}=$ eric $\& A N=\mathrm{E}$ J921197\&lang $=$ es\&site $=$ ehost-live

Onrubia, J. (2016). Aprender y enseñar en entornos virtuales: actividad conjunta, ayuda pedagógica y construcción del conocimiento. RED. Revista de Educación a Distancia, 50, 1-14. Recuperado de http://www.um.es/ead/red/50/onrubia.pdf

ONU. (1948). Declaración Universal de Derechos Humanos. Resolución de la Asamblea General 217 A del 10 de diciembre de 1948.

ONU. (1999). Aplicación del pacto internacional de derechos económicos, sociales y culturales. Observaciones generales 13.

PAL. (2016). Estadística de apertura de entornos virtuales de aprendizaje 2016. Documento interno.

Pérez, R., López, R., Romero, D. y Urquía, E. (2016). E learning: Un proyecto de docencia virtual como proceso de enseñanza-aprendizaje apoyado en la red. Recuperado de http://eprints.sim.ucm. es/36183/1/Memoria\%20PIMCD\%20175.pdf

Pubiales, B. y de Fonseca, A. (2014). La formación universitaria en iberoamérica desde lo virtual, como entorno para la calidad pedagógica en la educación superior. Tendencias: Revista De La Facultad De Ciencias Económicas Y Administrativas, 15(1), 268286. Recuperado de http://search.ebscohost.com/login.aspx?direct $=$ true $\& \mathrm{db}=$ fua $\& A N=98656848 \&$ lang $=$ es\&site=ehost-live

Rama, C. (Ed.). (2012). La reforma de la virtualización de la universidad, el nacimiento de la educación digital. Recuperado de http://virtualeduca.org/documentos/observatorio/libro_la-reforma-de-la-virtualizacion-de-la-universidad-claudio-rama-udg-2012.pdf

Rama, C. (2013). Los caminos de las reformas. La virtualización universitaria en América Latina. Revista historia de la educación latinoamericana, 14(19). Recuperado de http://revistas.uptc.edu. co/revistas/index.php/historia_educacion_latinamerican/article/ view/1985

Ramírez, F. y Rama, C. (Eds.) (2014). Los recursos de aprendizaje en la educación a distancia, nuevos escenarios, experiencias y tendencias. Recuperado de http://www.virtualeduca.org/documentos/ observatorio/2014/los-recursos-de-aprendizaje.pdf

Rodríguez, G., Gómez, V. y Ariza, M. (2014). Calidad de la educación superior a distancia y virtual: un análisis de desempeño 
académico en Colombia. Investigación Y Desarrollo, 22(1), 79119. Recuperado de http://search.ebscohost.com/login.aspx?direct=true \&db=fua\&AN=95806031\&lang=es\&site=ehost-live

Sánchez, A. (2013). Calidad y accesibilidad del elearning: encuentro y reflexión desde una perspectiva inclusiva. Revista Digital de Investigación Educativa. Recuperado de http://www.revistaconecta2.com.mx/archivos/revistas/revista8/8_4.pdf

Tilve, F., Núñez, Á. y Fernández, R. (2013). E-Learning: Otra manera de enseñar y aprender en una universidad tradicionalmente presencial. Estudio de caso particular. Profesorado. Revista de $\mathrm{Cu}$ rrículum y Formación del Profesorado, 17(3), 19. Recuperado de http://recyt.fecyt.es/index.php/profesorado/article/view/41768

UNED. (2012). Plan de Desarrollo Académico 2012-2017. Vicerrectoría Académica. Recuperado de http://www.uned.ac.cr/academica/plan_academico/bienvenida.html

UNED. (s.f.). Rectoría: misión y visión. Recuperado de http://www. uned.ac.cr/rectoria/myv

UNED. (2017). Consideraciones para el diseño y oferta de asignaturas en línea. Documento interno.

Vargas, G. (2014). Calidad y equidad de la educación superior pública. Aspectos por considerar en su interpretación. Revista Educación, 31(2), 11-27. Recuperado de http://revistas.ucr.ac.cr/index.php/ educacion/article/view/1241 